\title{
RECONHECIMENTO DE GESTOS DINÂMICOS PARA A INTERAÇÃO HUMANO-ROBÔ
}

\author{
Clebeson Canuto do Santos, Jorge Leonid Aching Samatelo, \\ Raquel Frizera Vassallo \\ Departamento de Engenharia Elétrica, Universidade Federal do \\ Espírito Santo, Av. Fernando Ferrari, 514, Goiabeiras, Vitória - ES , \\ Cер: 29075-910 \\ (e-mails: clebeson.canuto@gmail.com,jorge.samatelo@ufes.br, \\ raquel@ele.ufes.br)
}

\begin{abstract}
With the advance of technologies, machines are closer to people. Thus, it is necessary to develop interfaces, like gestures, capable of providing an intuitive way of interaction. Therefore, this work proposes a modification of the Star RGB technique, which condenses the temporal information of a video in just one RGB image. The proposal, called Star RGB+, applies the Star RGB technique over the channels of a video. So, rather than only one RGB image, this proposes yields three images as a condensed representation of a gesture in an RGB video clip. As complement, still is proposed an ensemble-like architecture using 3 VGG16, as feature extractor, one for each image, and a fully connected architecture as classifier that recieves the fused information came out from the extractors. The main experiments were carried out on GRIT (Gesture Commands for Robot inTeracton) dataset, used for human-robot interaction, and achieve more than $97 \%$ of accuracy, precision, recall and f1-score, outperforming the author's original results in more than $5 \%$ for every metric. In order to compare results with the original propose of Star RGB, a secondary experiment was carried out on Montalbano dataset, achieving $92.34 \%$ of accuracy, outperforming the autor's results in more than $9 \%$. This shows the contribution of this work for dynamic gesture recognition field, mainly for those ones used for human-robot interaction.

Resumo: Com o avanço das tecnologias, as máquinas estão cada vez mais próximas das pessoas. Assim, é necessário desenvolver interfaces, como gestos, que forneçam uma maneira intuitiva de interação entre humano e robôs. Neste sentido, este trabalho visa propor uma modificação na técnica Star RGB, que condensa a informação temporal de um vídeo em apenas uma imagem RGB. A proposta aqui apresentada, chamada Star RGB+, aplica a técnica Star RGB nos canais de cor de um vídeo. Sendo assim, ao invés de apenas uma imagem RGB, esta proposta produz três imagens como representação condensada de um gesto presente em um vídeo colorido. Como complemento, é proposta também uma arquitetura do tipo ensemble utilizando para isso três redes VGG16 pretreinadas, uma para cada imagem, como extrator de características e uma arquitetura totalmente conectada como classificador que recebe a combinação das características extraídas por cada VGG16. Os principais experimentos foram realizados na base de dados GRIT (Gesture Commands for Robot in Teracton), usada para interação homem-robô, e atingiram mais de $97 \%$ em todas as métricas, acurácia, precisão, recall e F1-score, superando os resultados originais dos autores em mais de $5 \%$ em todas elas. A fim de comparar a melhora da proposta em relação à original, um experimento secundário foi realizado na base de dados Montalbano, alcançando $92,4 \%$ de taxa de reconhecimento, superando os resultados dos autores em mais de $9 \%$. Isso mostra a contribuição deste trabalho para o reconhecimento de gestos dinâmicos, principalmente para aqueles destinados à interação humano-robô.
\end{abstract}

Keywords: dynamic gesture recognition; human-robot interaction; artificial neural networks; temporal information condensation

Palavras-chave: reconhecimento de gestos dinâmicos; interação humano-robô; redes neurais artificiais; condensação de informação temporal. 


\section{INTRODUÇÃO}

Desde o advento do Microsoft Kinect ${ }^{1}$ em meados de 2011, existe uma forte tendência da utilização de sensores multimodais para o reconhecimento de gestos dinâmicos. Isto, devido à possibilidade de captura do esqueleto e dados de profundidade de um individuo presente no ambiente interacional, os quais oferece informações resumidas e precisas sobre o movimento da mesma. Assim, diferentes trabalhos têm utilizado tais sensores a fim de melhorar a taxa de reconhecimento dos gestos. A esse variado conjunto de informações é dado o nome de multimodal, e podem servir de entrada para um único modelo, como aquele apresentado em (Neverova et al., 2016).

Trabalhos que utilizam dados multimodais, obtêm resultados satisfatórios quando considerando apenas a taxa de acerto do reconhecedor. No entanto, muitos deles desconsideram que a maioria dos possíveis ambientes interacionais não contam a priore com tais sensores. Dessa maneira, quando recebem com entrada apenas informações de cor, comumente encontradas em ambientes mais gerais, fornecem resultados inferiores àquelas concentram esforços no uso apenas desse tipo de informação, como é o exemplo de santos et al. (2018a) e santos et al. (2018b).

Um dos grandes problemas que faz com que as informações de esqueleto e profundidade sejam preferidas em comparação à informação de cor é a dificuldade em relacionar o mudança de intensidade de cada pixel durante a execução do gesto. Assim, surgem abordagens que tentam aplicar filtros sobre uma sequência de frames de um vídeo, a fim de, juntamente com a informação de fluxo ótico, capturar as informações de movimento e contexto nos quais determinada ação é executada. Aqui, fala-se em ação pois é a grande área onde os gestos dinâmicos estão inseridos. Tais abordagens são comumente chamadas de fluxo duplo (two stream), sendo (Simonyan and Zisserman, 2014a), (Feichtenhofer et al., 2016) alguns dos principais trabalhos sobre o assunto. O problema desse tipo de solução é a necessidade de definição de um tamanho de janela sobre a qual o filtro é aplicado. Pois, janelas grandes ou pequenas acabam por beneficiar gestos de curta ou longa duração, respectivamente. Assim, resultados obtidos por tais soluções estão fortemente relacionadas ao comprimento dos vídeos que compõem a base de dados utilizada no treinamento. Diminuindo-se assim, as chances de sucesso em quando aplicados em problemas mais diversos.

Uma abordagem diferente para o reconhecimento de gestos dinâmicos em vídeos foi adotada por Tsironi et al. (2017), onde os autores utilizaram uma Rede Neural Convolucional (CNN - Convolutional Neural Network) para extrair características da diferença absoluta entre duas imagens consecutivas, e um classificador baseado em uma rede neural recorrente LSTM (Long-Short Time Memory) para poder capturar a informação temporal entre as mesmas. Além de ser uma solução interessante, outra contribuição importante dada pelo trabalho foi a disponibilização de uma base de dados de gestos dinâmicos de corpo inteiro utilizados na Interação Humano-Robô (Human-Robot In-

\footnotetext{
^ Reconhecimento à CAPES pelo suporte financeiro dado por meio da Bolsa de Doutorado concedida ao primeiro autor.

1 https://msdn.microsoft. com/en-us/library/hh438998.aspx
}

teraction - HRI). Uma vez que, segundo os próprios autores, além da grande parte das bases de dados de gestos serem destinados ao gestos estáticos, aqueles destinados ao gestos dinâmicos, devido a serem utilizados na interação humano-computador, são, em sua maioria, focados apenas nos movimentos das mãos. Assim, uma base de dados que contém o registro dos indivíduos de corpo inteiro são de importância para o desenvolvimento da interação natural entre humanos e robôs.

Outros trabalhos que também objetivam o reconhecimento de gestos dinâmicos é (santos et al., 2018a,b). Ambos propuseram duas formas de representar um gesto em um vídeo na forma condensada de uma imagem RGB. A diferença principal entre as duas abordagens é que enquanto a primeira usa apenas a informação de intensidade entre imagens a segunda usa a informação de cor. Apesar de ambos abordagens serem efetivas considerando os resultados apresentados, a segunda abordagem é consideravelmente melhor que a primeira. A base de dados utilizada para a avaliação de ambas as propostas foi a Montalbano (Escalera et al., 2013), a qual não é destinada para a interação humano-robô e sim para para interação humano-humano utilizando, especificamente, um vocabulário de gestos emblemáticos italianos. No entanto, são abordagens muito interessantes e merecem ser aplicadas em bases como a disponibilizada por Tsironi et al. (2017).

Dessa maneira, este trabalho propõe modificações sobre a proposta de santos et al. (2018b), a fim de oferecer outra alternativa para a utilização de cor dos vídeos, assunto principal em (santos et al., 2018a), além de aplicar a solução na base de dados GRIT (Tsironi et al., 2017), destinada à interação humano-robô. Com isso, deseja-se obter resultados melhores que os apresentados em (santos et al., 2018b) e (santos et al., 2018a), além de contribuir para a interação humano-robô por meio da sua aplicação em uma base de dados destinada a tal propósito.

Sendo assim, o presente manuscrito está estruturado da seguinte maneira: a Seção 2 traz os trabalhos relacionados ao tema em discussão; na Seção 3 é descrita a proposta principal; a Seção 4 apresenta experimentos realizados; a Seção 5 discute os resultados obtidos por meio dos experimentos e; finalmente, na Seção 6 são expostas as conclusões e os trabalhos futuros.

\section{TRABALHOS RELACIONADOS}

O reconhecimento de gestos dinâmicos é um campo de pesquisa que, mesmo ainda pouco explorado, vem despertando grande interesse nos últimos anos. Isso devido ao desejo de desenvolver uma interface para interagir com máquinas e outros dispositivos de maneira intuitiva. No entanto, como um gesto pode ser representado de diferentes formas dentro de um mesmo contexto, reconhecer claramente um gesto dinâmico é um problema com um alto nível de dificuldade. Além disso, a maneira como os gestos são realizados depende não apenas da sequência de movimentos corporais, mas também do aspecto cultural das pessoas que os empregam. Consequentemente, ainda há muito trabalho a ser feito para conseguir uma interface capaz de fornecer uma comunicação efetiva entre humanos e máquinas com base em gestos. 
Por essa razão, base de dados bem estruturadas como o Montalbano são utilizadas como forma de contribuir para o problema. No entanto, além de não ser destinada ao problema do HRI, a sua aquisição foi realizada utilizando o sensor Microsoft Kinect 360. Assim, os dados de natureza multimodal (cor, profundidade, esqueleto, áudio e máscara de usuário) fazem com que os principais trabalhos, como (Li et al., 2017), (Joshi et al., 2017), (Efthimiou et al., 2016), (Neverova et al., 2016), concentrem-se no uso da multimodalidade dos dados, quase que descartando a possibilidade do uso apenas de informação de cor. Isso, como discutido antes, pode condicionar seu uso apenas em ambientes possuidores de tais sensores, o que não acorre na maioria dos ambientes, onde as câmeras RGB são facilmente encontradas.

Mesmo assim, devido às suas características de tamanho (mais de 13.000 gestos) e forma de captura (gestos de corpo inteiro), santos et al. (2018b,a) propuseram uma abordagem que condensa cada vídeo em apenas uma imagem e aplicaram a proposta nos vídeos da base. santos et al. (2018b) propuseram uma bordagem em que cada vídeo em tons de cinza é dividido em três partições e em cada uma delas é calculada a soma do valor absoluto da diferença entre dois frames consecutivos, como mostrado na Equação (1). No trabalho os autores chamam a abordagem de representação estrela, no entanto, aqui, a mesma será chamada de Star RGB.

$$
\mathbf{M}(i, j)=\sum_{k=2}^{N}\left|\mathbf{I}_{k-1}(i, j)-\mathbf{I}_{k}(i, j)\right|,
$$

onde: $\mathbf{M}$ é a representação resultante; $\mathbf{I}_{k}$ é o $k$-ésimo frame em escala de cinza do vídeo de $N$ frames contendo uma parte do gesto; $(i, j)$ é a posição de um pixel na sequência de vídeo; | • | é o operador valor absoluto.

Já em (santos et al., 2018a), a proposta foi ligeiramente modificada para que a informação de cor do vídeo seja levada em consideração, uma vez que a Equação (1) espera receber duas imagens em tons de cinza. Com isso, a proposta foi a de utilizar uma reformulação da distância cosseno (Equações (2) e (3)) entre duas imagens RGB consecutivas ao invés da diferença absoluta. Assim, os autores mostraram que a nova proposta (Equação (4)) gerou uma melhora considerável dos resultados, pois, enquanto que em (santos et al., 2018b) alcançou-se uma acurácia de $83.10 \%$ em (santos et al., 2018a) alcançou-se $89.75 \%$ na base de dados Montalbano.

$$
\begin{gathered}
\lambda=1-\cos (\theta)=1-\frac{\mathbf{I}_{k-1}(i, j)^{T} \mathbf{I}_{k}(i, j)}{\left\|\mathbf{I}_{k-1}(i, j)\right\|_{2}\left\|\mathbf{I}_{k}(i, j)\right\|_{2}} . \\
\mathbf{D}(i, j)=\left(1-\frac{\lambda}{2}\right) \cdot\left\|\mathbf{I}_{k-1}(i, j)\right\|_{2}-\left\|\mathbf{I}_{k}(i, j)\right\|_{2} \mid, \\
\mathbf{M}(i, j)=\sum_{k=2}^{N} \mathbf{D}(i, j) .
\end{gathered}
$$

Com isso em mente, este trabalho propõe mudanças sobre a representação em (santos et al., 2018b), a fim de utilizar a informação de cor de cada vídeo utilizando a diferença absoluta entre frames (Equação (1)), ao invés da distância cosseno, apresentada em (santos et al., 2018a). Para isso, o Star RGB será aplicado a cada um dos três canais de cor de um vídeo. O que resultará em três imagens RGBs distintas, uma para cada canal. Cada imagem será dada como entrada a uma CNN do tipo VGG16, o que formará um conjunto (ensemble) de três CNNs. As saídas das CNNs serão fundidas utilizando um operador de média. Logo após, será dada com entrada para uma rede totalmente conectada responsável por classificar o gesto presente no vídeo e representado na forma das 3 imagens RGBs. Esta nova proposta será chamada aqui de Star RGB+.

Acredita-se que tanto as alterações propostas para a representação Star RGB, quanto a arquitetura de classificação em ensemble que se propõe podem ser consideradas as principais contribuições deste trabalho.

\section{PROPOSTA}

Nesta seção será descrita a técnica de reconhecimento de gestos proposta, a qual está baseada em duas etapas principais, especificamente:

- pré-processamento: cada vídeo de entrada é representado na forma de três imagens RGBs usando uma versão modificada da Star RGB.

- classificação: um classificador dinâmico de gestos é treinado usando um conjunto (ensemble) de CNNs. Especificamente, as três imagens coloridas, resultantes da etapa de pré-processamento, são fornecidas como entrada para três CNNs pré-treinadas. Seguidas por uma camada totalmente conectada e um classificador softmax que dá como saída a probabilidade do vídeo, representado pelas das três imagens, pertencer a cada um dos gestos possíveis.

Para melhor compreensão, ambos os passos estão especificados a seguir.

\subsection{Pré-processamento}

Um dos problemas com a Star RGB é que ela não leva em consideração as informações de cor (Matiz e saturação) de cada frame do vídeo, uma vez que o processo é calculado usando apenas sua representação em escala de cinza (intensidade). Assim, o objetivo aqui é representar a informação temporal presente em um vídeo colorido usando uma melhoria da representação Star RGB. Essa melhoria (Star RGB+) é calculada em duas etapas, sendo a primeira etapa proposta neste trabalho e segunda utilizando a proposta original apresentada em (santos et al., 2018b), a saber:

- cada vídeo colorido contendo um gesto dinâmico completo terá os seus canais separados, resultando em 3 vídeos em tons de cinza.

- aplica-se a proposta em (santos et al., 2018b) (ilustrada na Figura 1), em cada um dos 3 vídeos obtidos na etapa anterior (Figura 2). Assim, como resultado ter-se-á 3 imagens coloridas, uma para cada um dos 3 canais ( $\mathrm{R}, \mathrm{G}$ e $\mathrm{B})$ do vídeo contendo o gesto. 


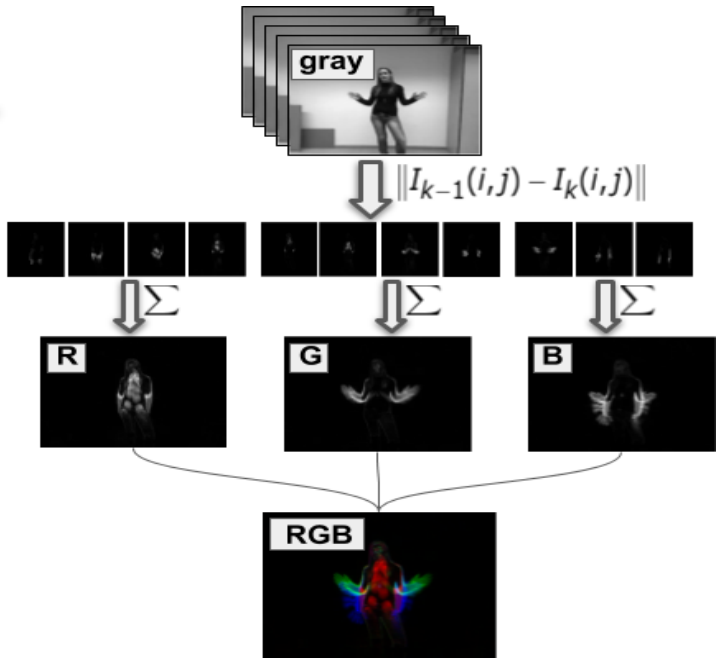

Figura 1. Star RGB: representação de um gesto dinâmico proposto em (santos et al., 2018b).

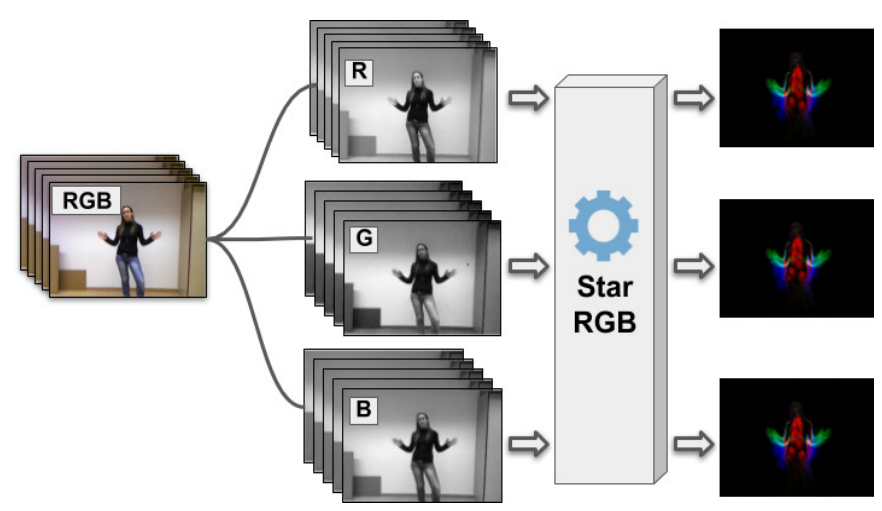

Figura 2. Processo de aplicação do Star RGB+ sobre um vídeo.

\subsection{Classificação}

Assim como em (santos et al., 2018b), a CNN utilizada aqui, base para o ensemble proposto na arquitetura principal, será a VGG16 (Simonyan and Zisserman, 2014b), especializada na classificação de imagens, previamente treinadas no banco de dados ImageNet. Essa base de dados foi disponibilizada na competição ILSVRC-2014 e contém mais de 1,2 milhões de imagens distribuídas entre 1000 categorias distintas (Russakovsky et al., 2015). A arquitetura proposta é mostrada na Figura 3 e explicada na continuação.

Como o passo de pré-processamento gera três imagens que representam cada gesto, um conjunto de três VGG16 pré-treinadas são usadas. Assim, cada uma das imagens é dada como entrada para uma VGG16 composta pelas suas 8 primeiras camadas convolucionais. A junção das saídas de cada uma das três redes (chamada de ensemble) é realizada utilizando uma operação de média por meio de uma camada de Average Pooling. O resultado é dado como entrada para uma camada completamente conectada de 3.072 neurônios ocultos seguidos por outra de 9 neurônios, sendo a primeira ativada com a função ReLU e a segunda pela função softmax. Esta última camada é responsável por prever a qual das 9 classes de gestos (número de classes de gestos contidas na base usada - Seção 4) pertence o vídeo representado pelas 3 imagens de entrada.

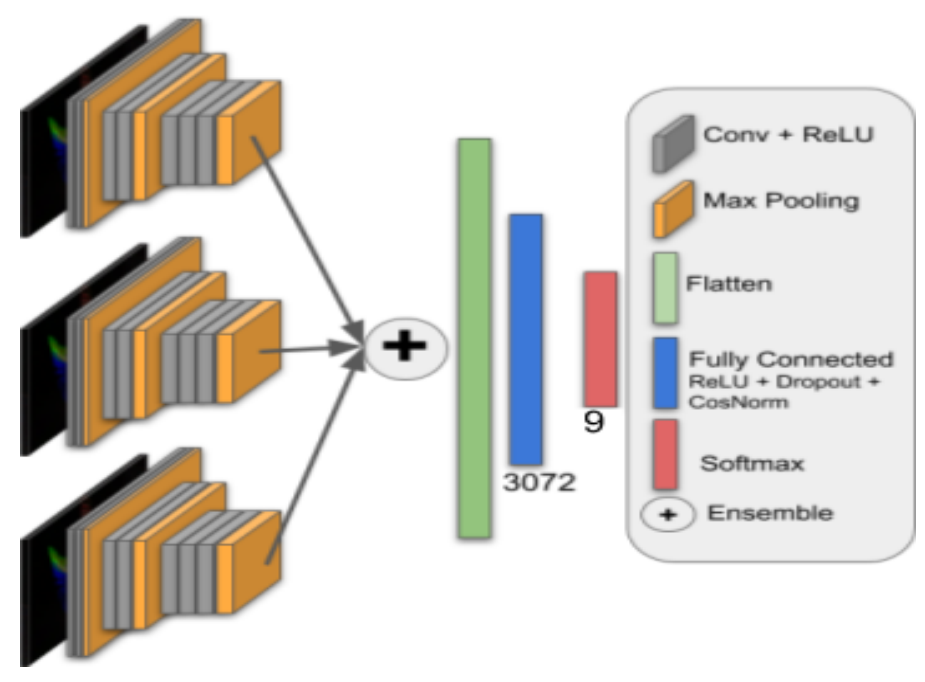

Figura 3. Arquitetura proposta baseada na rede VGG16.

A fim de diminuir o efeito do deslocamento da variância discutido em (LeCun et al., 1998), foi utilizado o cosine normalization (Ioffe and Szegedy, 2015), que garante que a distribuição dos pesos mantenha-se entre $[-1,1]$. O mesmo foi aplicado entre as camadas totalmente conectadas e serviu como uma alternativa mais eficiente, do ponto de vista de processamento, ao tradicional batch normalization (Ioffe and Szegedy, 2015).

Ao usar três redes como extrator de características, o número de parâmetros do modelo acaba crescendo consideravelmente. Dessa forma, é essencial usar uma técnica de regularização apropriada para diminuir a capacidade de sobre ajuste da rede. Nesse sentido, após vários testes usando os regularizadores $L 1, L 2$ e dropout (Srivastava et al., 2014), O L1 em conjunto com o dropout foram os que trouxeram os melhores resultados. Sendo o $L 1$ aplicado na função de custo (Entropia Cruzada) o operador de dropout inserido entre as duas camadas totalmente conectadas.

A melhora conseguida com dropout deve-se a sua capacidade de diminuir a variância do modelo (Srivastava et al., 2014). Já o regularizador $L 1$, por forçar a esparsidade dos pesos da rede, tem a capacidade de reduzir a complexidade das amostras quando a mesma possui muitas características irrelevantes $(\mathrm{Ng}, 2004)$. Aqui, considera-se como características irrelevantes os pixels das imagens Star RBG+ que assumem valores próximos a zero e que correspondem ao fundo da cena, o que pode ser observado na Figura 1. Em media, cada Star RGB+ tem $42.47 \%$ dos seus pixels ( $p$ ) com valor próximo de zero $(p \leq 5, p \in[0,255])$.

\section{EXPERIMENTOS}

A base de dados GRIT (Gesture Commands for Robot inTeracton) (Tsironi et al., 2017) foi utilizada para o treinamento e avaliação da arquitetura proposta. Essa base de dados é composta por 543 gestos, distribuídos em 9 classes distintas. A Figura 4 mostra uma representação dos gestos contidos na mesma. Em complemento, a Figura 5 mostra a representação Star RGB+ de uma amostra de cada um dos 9 diferentes gestos. A sua escolha deveu-se ao 
fato dela ser uma das únicas bases de dados que propõem gestos dinâmicos de corpo inteiro para a interação humanorobô e, uma importante característica, baseados apenas em informação de cor.

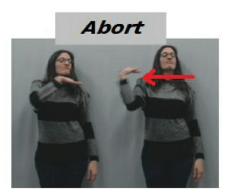

No!
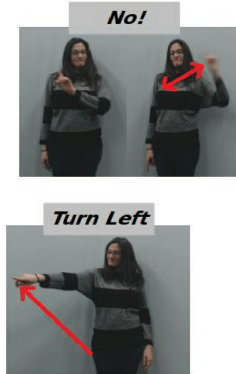
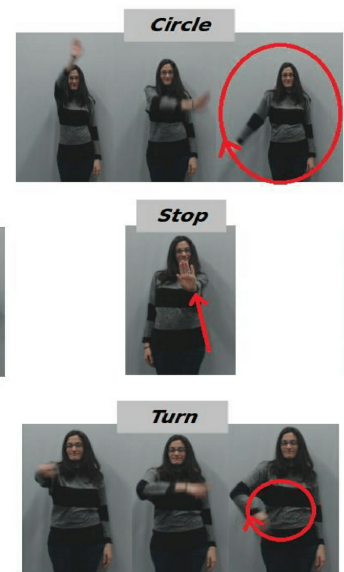

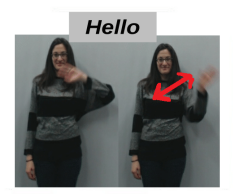

Warn

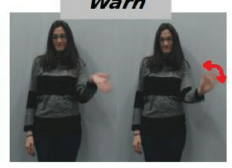

Turn Right

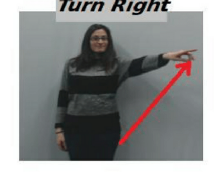

Figura 4. Representação dos nove gestos presentes na base de dados GRIT (Tsironi et al., 2017).

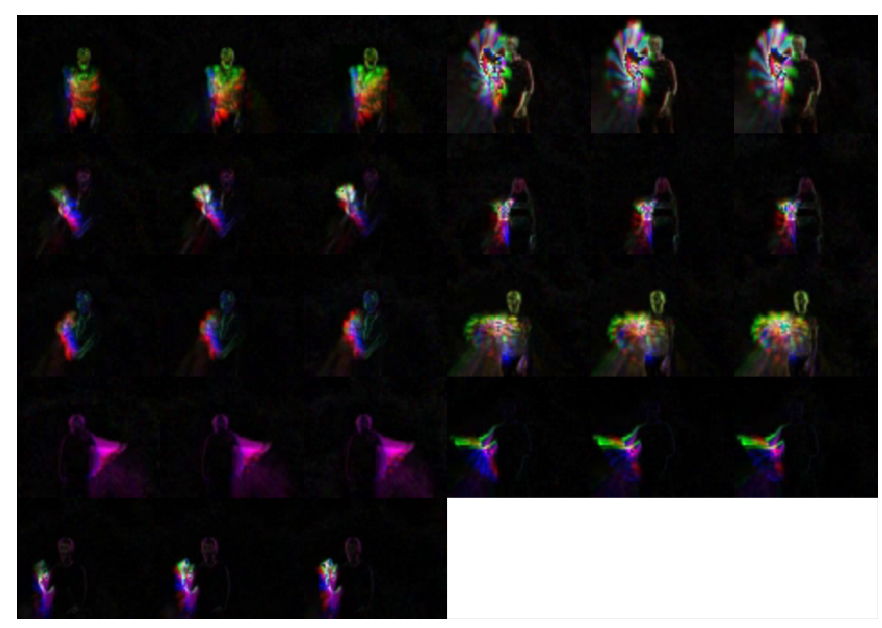

Figura 5. As três imagens do Star RGB+ para uma amostra de cada gesto da base de dados GRIT. As imagens estão dispostas na mesma ordem que a Figura 4.

\subsection{Implementação e Treino}

Para realizar o pré-processamento, armazenamento e carregamento dos dados, utilizou-se a linguagem Python e sua biblioteca numérica Numpy. O modelo Proposto foi implementado e treinado utilizando o framework Pytorch na sua versão 1.0: software de código aberto desenvolvido pelo grupo de pesquisa de inteligência artificial do Facebook ${ }^{2}$ para o aprendizado de máquina (Paszke et al., 2017).

Os experimentos foram realizados em um computador com a seguinte configuração: (i) Sistema Operacional Linux, distribuição Ubuntu 16.04, versão servidor; (ii) Processador Intel Core i7-7700, 3.60GHz com 4 núcleos físicos; ( iii) 32GB de RAM; (iv) 1TB de unidade armazenamento permanente (disco rígido); $(v)$ Placa gráfica Nvidia Titan $V$, com $12 \mathrm{~GB}$ de memória dedicada.

\footnotetext{
2 https://research.fb.com/category/facebook-ai-research/
}

Durante a etapa de treinamento do classificador foram utilizados os seguintes hiperparâmetros: tamanho do batch de 96, um máximo de 50 épocas, algorítimo de otimização Adam com taxa de aprendizado de 5 e-3 decaindo $5 \%$ a cada época, e uma taxa de dropout de 0.5.

Para poder comparar os resultados obtidos com aqueles apresentados em Tsironi et al. (2017), os experimentos foram realizados seguindo o mesmo processo utilizado pelos autores. $\mathrm{O}$ qual consiste na realização de cinco etapas de hold out, sendo que a cada uma delas os 543 vídeos dever ser embaralhados e divididos em duas partes: $80 \%$ para treino e $20 \%$ para teste.

Outra forma de regularização utilizada foi o método de parada antecipada (early stop). Dessa forma, foi possível fazer com que o treinamento pudesse ser interrompido quando a taxa média de acerto das últimas cinco épocas do treinamento ultrapasse os $99 \%$.

$\mathrm{Na}$ avaliação, o desempenho de um modelo de reconhecimento de gestos multi-classe normalmente é ponderado pela acurácia média entre classes. No entanto, como o processo de treino e teste se deu em cinco etapas de hold out, o desempenho do mesmo foi medido por meio de 4 métricas todos os cinco resultados, assim como feito no trabalho original, a saber: acurácia, precisão, recall e F1score.

\section{RESUlTADOS E DISCUSSÕES}

Usando a arquitetura proposta, após uma média de 20 épocas entre cada etapa de treinamento, os resultados das métricas estão apresentados na Tabela 1. Como pode ser notado, esta proposta alcançou os melhores resultados para a base de dados GRIT(Tsironi et al., 2017), chegando a superar os resultados apresentados pelos autores. A melhoria foi de cerca de $5 \%$ para todas as métricas: acurácia, precisão, recall e F1-score. Além disso, o melhor resultado alcançado pela proposta foi de $99,08 \%$ de acurácia contra $92,59 \%$ obtidos pelos autores. Isso é um indicativo de que a nova representação Star RGB+ é importante para o reconhecimento de gestos dinâmicos destinados à interação humano-robô.

Tabela 1. Comparação dos resultados obtidos pela presente proposta em relação aos apresentados em (Tsironi et al., 2017) usando a base de dados GRIT.

\begin{tabular}{lll} 
Métrica & (Tsironi et al., 2017) & Proposta \\
\hline Acurácia & $91.67 \% \pm 1.13 \%$ & $97.51 \% \pm 1.17 \%$ \\
Precisão & $92.35 \% \pm 0.98 \%$ & $97.59 \% \pm 1.07 \%$ \\
Recall & $91.90 \% \pm 1.05 \%$ & $97.40 \% \pm 1.18 \%$ \\
F1-score & $92.13 \% \pm 1.00 \%$ & $97.25 \% \pm 1.16 \%$ \\
\hline
\end{tabular}

Mesmo que os resultados tenham sido satisfatórios, ainda não se conseguiu medir a melhora da proposta em relação às duas abordagens anteriores (santos et al., 2018b,a), uma vez que elas não apresentaram resultados para a base de dados GRIT. Sendo assim, a fim de realizar tal comparação, a arquitetura proposta foi aplicada sobre a base de dados Montalbano, obtendo uma acurácia de 92.34\%. Como já era esperado, esse resultado é muito superior aos $83.10 \%$ apresentado em (santos et al., 2018b) e ainda melhor que os $89.75 \%$ apresentado em (santos et al., 2018a). Isso mostra a superioridade da proposta em relação às demais comparadas. 
Sendo assim, após a exposição dos resultados alcançados, percebe-se que a proposta aqui apresentada tanto pode ser utilizada em bases de dados de gestos dinâmicos que objetivam a interação humano robô, principal objetivo deste trabalho, como em outras, como a Montalbano, que obetivam a interação humano-humano. Não restando dúvidas quanto a sua considerável contribuição para essa área de estudos, que, mesmo com todo o desenvolvimento do deep learning, ainda carece da atenção da comunidade acadêmica.

\section{CONCLUSÕES E TRABALHOS FUTUROS}

Apesar de nos últimos anos os estudos sobre reconhecimento de imagens tenham crescido substancialmente devido ao uso de arquiteturas de redes neurais cada vez mais profundas, o reconhecimento de gestos dinâmicos não tem acompanhado tal crescimento. Além disso, os principais trabalhos recentes estudam soluções que usam dados multimodais fornecidos por sensores (por exemplo, o Microsoft Kinect) dificilmente encontrados em ambientes interacionais, nos quais as câmeras RGBs são dominantes. Com isso, diminuem-se as chances de sucesso na implantação de tais soluções em ambientes mais gerais onde a interação humano-robô se faz necessária.

Dessa maneira, este trabalho propôs uma modificação significativa sobre a proposta em (santos et al., 2018b) a fim de reconhecer gestos dinâmicos destinados à interação humano-robô presentes na base de dados GRIT(Tsironi et al., 2017). Tal proposta cosiste de uma arquitetura baseada num ensemble de três CNNs do tipo VGG16, que tem a tarefa de classificar o gesto representado em 3 imagens RGBs obtidas a partir da condensação da informação temporal de um vídeo.

Após os experimentos, a proposta aqui apresentada alcançou resultados melhores que aqueles fornecidos pelos autores da base de dados, mais de $5 \%$ em todas as métricas de avaliação, além de ultrapassar significativamente os resultados em (santos et al., 2018b,a) após aplicada sobre a base de dados Montalbano(Escalera et al., 2013). Com isso, confirma-se a importância da proposta apresentada para o reconhecimento de gestos dinâmicos, principalmente para aqueles destinados à interação humano-robô.

Mesmo com todas as melhoras adquiridas por este trabalho, propõe-se como trabalhos futuros a utilização de outras CNNs como base para o ensemble, além da aplicação da arquitetura em um ambiente interacional real que utilize gestos dinâmicos como forma de comunicação entre humanos e robôs.

\section{REFERÊNCIAS}

Efthimiou, E., Fotinea, S.E., Goulas, T., Koutsombogera, M., Karioris, P., Vacalopoulou, A., Rodomagoulakis, I. Maragos, P., Tzafestas, C., Pitsikalis, V., Koumpouros, Y., Karavasili, A., Siavelis, P., Koureta, F., and Alexopoulou, D. (2016). The MOBOT rollator human-robot interaction model and user evaluation process. 2016 IEEE Symposium Series on Computational Intelligence (SSCI), 1-8. doi:10.1109/SSCI.2016.7850061.

Escalera, S., Gonzàlez, J., Baró, X., Reyes, M., Lopes, O., Guyon, I., Athitsos, V., and Escalante, H. (2013). Multimodal gesture recognition challenge 2013: Dataset and results. In Proceedings of the 15th ACM on International conference on multimodal interaction, 445-452. ACM.

Feichtenhofer, C., Pinz, A., and Zisserman, A. (2016). Convolutional two-stream network fusion for video action recognition. In Proceedings of the IEEE conference on computer vision and pattern recognition, 1933-1941. doi:10.1109/CVPR.2016.213.

Ioffe, S. and Szegedy, C. (2015). Batch normalization: Accelerating deep network training by reducing internal covariate shift. arXiv preprint arXiv:1502.0316\%.

Joshi, A., Monnier, C., Betke, M., and Sclaroff, S. (2017). Comparing random forest approaches to segmenting and classifying gestures. Image and Vision Computing, 58, 86-95. doi:10.1016/j.imavis.2016.06.001.

LeCun, Y., Bottou, L., Orr, G.B., and Müller, K.R. (1998). Efficient backprop. In Neural networks: Tricks of the trade, 9-50. Springer.

Li, C., Hou, Y., Wang, P., and Li, W. (2017). Joint Distance Maps Based Action Recognition with Convolutional Neural Networks. IEEE Signal Processing Letters, 24(5), 624-628. doi:10.1109/LSP.2017.2678539.

Neverova, N., Wolf, C., Taylor, G., and Nebout, F. (2016). Moddrop: adaptive multi-modal gesture recognition. IEEE Transactions on Pattern Analysis and Machine Intelligence, 38(8), 1692-1706.

Ng, A.Y. (2004). Feature selection, 11 vs. 12 regularization, and rotational invariance. In Proceedings of the twenty-first international conference on Machine learning, 78. ACM.

Paszke, A., Gross, S., Chintala, S., Chanan, G., Yang, E., DeVito, Z., Lin, Z., Desmaison, A., Antiga, L., and Lerer, A. (2017). Automatic differentiation in pytorch.

Russakovsky, O., Deng, J., Su, H., Krause, J., Satheesh, S., Ma, S., Huang, Z., Karpathy, A., Khosla, A., Bernstein, M., et al. (2015). Imagenet large scale visual recognition challenge. International Journal of Computer Vision, $115(3), 211-252$.

santos, C.C., Samatelo, J.L.A., and Vassallo, R.F. (2018a). Improving Dynamic Gesture Recognition by Using CNNs and Color Information Representation. In $X I V$ Workshop de Visão Computacional, WVC.

santos, C.C., Silva, L.A., Queiroz, Felippe Mendonça, P.R., Samatelo, J.L.A., and Vassallo, R.F. (2018b). Uso de transfer learning para o reconhecimento de gestos dinâmicos. In XXII Congresso Brasileiro de Automática, $C B A$.

Simonyan, K. and Zisserman, A. (2014a). Two-stream convolutional networks for action recognition in videos. In Advances in neural information processing systems, $568-576$.

Simonyan, K. and Zisserman, A. (2014b). Very deep convolutional networks for large-scale image recognition. arXiv preprint arXiv:1409.1556.

Srivastava, N., Hinton, G., Krizhevsky, A., Sutskever, I., and Salakhutdinov, R. (2014). Dropout: A simple way to prevent neural networks from overfitting. The Journal of Machine Learning Research, 15(1), 1929-1958.

Tsironi, E., Barros, P., Weber, C., and Wermter, S. (2017). An analysis of convolutional long short-term memory recurrent neural networks for gesture recognition. Neurocomputing, 268, 76-86. 